Wo eine specielle Angabe fehlt, beziehen sich die Beobachtungen bei » $\mathbf{K}$ * auf das Passiren der Mitte, bei $\rightarrow$ M auf das gänzliche Verschwinden der einzelnen Flecke.

Pola, 1900 November.

Der Vorstand: I. von Benko.

\title{
Beobachtung der Saturnbedeckung am 13. Juni 1900
}

an der Sternwarte des Hydrographischen Amtes der k. und k. Kriegsmarine in Pola.

Infolge ungüstiger Luftverhältnisse und minderer Bilder konnte am Abend des 13. Juni 1900 keine so starke Vergrösserung angewendet werden, wie dies eine gute Beobachtung in Anbetracht des hellen Vollmondrandes erfordert hätte. Es gelang nur folgende vier Momente innerhalb der Fehlergrenze \pm 0.5 zu beobachten.

Eintritte des Mondostrandes:

Pola, 1900 November.
M. Z. Pola

I. Am äusseren Westrande des äussersten Ringes $10^{\text {h }} 45^{\mathrm{m}} 30^{\mathrm{s}}$ II. 》 $~ \gg$ Crape (C) 》 ro 4540 III. »Westrande der Saturnkugel 10 $455 \circ$ IV. "Ostrande 》 $\gg$ I0 4630

Es beobachtete Linienschiffsfähnrich E. Marchetti am 6-zöll. Refractor mit 100-facher Vergrösserung.

Der Vorstand: I. von Benko.

\section{Zur Leonidenerscheinung 1900.}

In Prag wurde das Phaenomen des Leonidenfalles vornehmlich von Herrn stud. astr. Anton Schlein, welcher den für längere Zeit beurlaubten ersten Assistenten aushülfsweise an der Sternwarte vertritt, beobachtet. Derselbe berichtet darüber :

Die Nacht vom I3. auf den I4. November I goo war in Prag in ihrer ersten Hälfte, von $6^{\mathrm{h}} 15^{\mathrm{m}}$ Abends angefangen, zu welcher Zeit sich die bislang bestandene Wolkendecke auflöste, bis I I $^{\text {h }} 30^{\mathrm{m}}$ Nachts sehr klar und heiter. Doch bald nach $13^{\mathrm{h}}$ zeigten sich im Osten Nebel, die in kurzer Zeit über den ganzen Himmel zogen und jede weitere Beobachtung unmöglich machten. Ich beobachtete von der 26 Meter über dem Erdboden gelegenen meteorologischen Terrasse der Sternwarte aus von ${ }_{1} \mathrm{I}^{\mathrm{h}} 3 \mathrm{O}^{\mathrm{m}}$ bis $\mathrm{I}^{\mathrm{h}}$ den nächtlichen Sternhimmel; doch zeigten sich bis zur Zeit der Umwölkung keine Meteore.

Die folgende Nacht vom 14. auf den I 5. November hatte in ihrer ersten Hälfte vollkommen bedeckten Himmel bis $12^{\mathrm{h}} 30^{\mathrm{m}}$. Sodann heiterte es sich grösstentheils auf und blieb bis gegen $16^{\text {b }}$, namentlich in den Zenithalgegenden, sehr klar. Ich beobachtete wieder von $13^{\mathrm{h}}$ an und notirte zu dieser Zeit 0.3 Bedeckung, die über dem Horizont als leichter, nebeliger Cirrostratus lagerte. Die Luft war mässig bis stark bewegt. Bis gegen $16^{\text {h }}$ beobachtete ich nun ununterbrochen, sah aber während der ganzen drei Stunden nur drei Meteore, die unstreitig den Leoniden angehörten und der Helligkeit nach Sternen 2. bis 3. Grösse gleichkamen. Sie zeigten sich in der Nähe des Zeniths und fielen innerhalb 5 Minuten. Im Folgenden sind die Zeiten ihres Sichtbarwerdens, die Richtung, aus der sie zu kommen schienen, der Weg, den sie zwischen den Gestirnen genommen, schliesslich annäherungsweise Rectascension und Declination ihres Anfangs- und Endpunktes auf Grund der 》Uebersichtskarte zur Beobachtung der Leoniden«, Epoche 1880, entworfen und bearbeitet von Dr. Karl Kostersitz, in welche die Einzeichnung geschah, gegeben.

I) Das erste Meteor wurde um $15^{\mathrm{h}} \mathrm{o}^{\mathrm{m}} \mathrm{M}$. Z. Prag sichtbar. Aus der Richtung des kleinen Löwen kommend, zwischen $\alpha$ Geminorum und $\beta$ Aurigae ziehend, hatte es als Anfangs punkt rund: $\alpha=8^{\mathrm{h}}$ und $\delta=+32^{\circ}$, als Endpunkt: $a=6^{\mathrm{h}} 30^{\mathrm{m}}$ und $\delta=+3^{\circ}$

2) Das zweite Meteor fiel einige Secunden nach diesem ersten. Es kam ebenfalls aus der Richtung des kleinen Löwen, $z o g$ aber nordwestlich an $\beta$ Aurigae vorüber. Die Coordinaten des Anfangspunktes waren ungefähr: $\alpha=8^{\mathrm{h}}, \delta=+40^{\circ}$, des Endpunktes: $\alpha=6^{\mathrm{h}} 20^{\mathrm{m}}, \delta=+48^{\circ}$.

3) Das dritte Meteor kam um $15^{\mathrm{h}} 5^{\mathrm{m}} \mathrm{M}$. Z. Prag aus derselben Richtung, wie die beiden vorangegangenen und zog zwischen $\alpha$ und $\gamma$ Orionis hindurch. Anfangspunkt: $\alpha=6^{\mathrm{h}}, \delta=+12^{\circ}$, Endpunkt: $\alpha=5^{\mathrm{h}}, \delta=+5^{\circ}$.

Nach $16^{\mathrm{h}}$ nahm die Bewölkung von Westen her zu, und nach kurzer Zeit war der ganze Himmel bedeckt.

In der nächsten Nacht vom $x_{5}$. auf den 16 . November beobachtete ich wieder von $14^{\mathrm{h}}$ bis $15^{\mathrm{h}}$ bei sehr klarem und heiterem Wetter. Doch zeigten sich keine Meteore mehr. «

Ich selbst hatte Auftrag gegeben, mich sofort $\mathrm{zu}$ benachrichtigen, falls sich in den fraglichen Nächten Bemerkens. werthes ereignen sollte. Am I 3 . November um $5^{\text {h }}$ sah ich nach dem Himmel; doch war es völlig trübe und aussichtslos. Am I 4. November um $134^{\text {h }}$ sah ich abermals vom Fenster meiner nach Süden gelegenen Wohnung aus nach dem Himmel, welcher theilweise klar erschien; Orion stand im Meridian. Durch fünf Minuten hindurch konnte indessen keine Stern. schnuppe wahrgenommen werden.

Zu Nr. 3683 , S. 225 der Astr. Nachr. wäre hach Vorstehendem zu berichtigen, dass in der Nacht vom 13. auf den 14. November nicht »stets der Himmel so verschleiert war, dass Sterne dritter Grösse nur eben noch sichtbar waren *; vielmehr war es hier am I 3 . November bis Mitternacht schön klar, so dass Herr Assistent Dörr von $10^{\mathrm{h}} 2_{2}^{\mathrm{m}}$ bis $12^{\mathrm{h}} 3^{\mathrm{m}}$. M. Z. Prag die Polhöhengruppe I, in welcher drei Sterne 7. Grösse vorkommen, mit dem Pistor \& Martin'schen Passageninstrument von nur $68 \mathrm{~mm}$ Oeffnung, vollständig beobachten konnte.

\section{Weinek.}

